\title{
One-Shot-Learning Gesture Recognition Using Motion History Based Gesture Silhouettes
}

\author{
Upal Mahbub a,", Tonmoy Roy ${ }^{a}$, Md. Shafiur Rahman ${ }^{a}$, Hafiz Imtiaz $^{a}$, Seiichi Serikawa ${ }^{b}$, Md. Atiqur Rahman Ahad \\ ${ }^{a}$ Department of Electrical and Electronic Engineering, Bangladesh University of Engineering and Technology, \\ Dhaka-1000, Bangladesh \\ ${ }^{\mathrm{b}}$ Kyushu Institute of Technology, 1-1 Sensui, Tobata, Kitakyushu 804-8550, Japan \\ ${ }^{c}$ Department of Applied Physics, Electronics \& Communication Engineering \\ University of Dhaka, Bangladesh
}

*Corresponding Author: atiqahad@univdhaka.edu

\begin{abstract}
A novel approach for gesture recognition based on motion history images is proposed in this paper for one- shot learning gesture recognition task. The challenge here is to perform satisfactory recognition operations with only one training example of each action, while no prior knowledge about actions, foreground/background segmentation, or any motion estimation and tracking are available. In the proposed scheme motion history imaging technique is applied to track the motion flow in consecutive frames. The information of motion flow is later utilized to calculate the percent change of motion flow for an action in different spatial regions of the frame. The space-time descriptor computed this way from the query video is a measure of the likeness of a gesture in a lexicon. Finally, gesture classification is performed based on correlation based and Euclidean distance based classifiers and the results are compared. Through extensive experimentations on a much diversified dataset the effectiveness of employing the proposed scheme is established.
\end{abstract}

Keywords: One-Shot-Learning, Gesture Recognition, Gesture Silhouettes.

\section{Introduction}

Human gesture recognition has its applicability in numerous real world domains, ranging from video surveillance to content-based video retrieval and video classification, control of home appliance to marshalling aircrafts, development of intelligent surveillance systems, and machine interaction with kids for game control to communication with deaf people through sign languages, etc. A human gesture recognition algorithm converts a high-bandwidth video into a compact description of the presence and movement of the human subjects in that scene and the recognition system helps a human user to control a variety of devices using gestures alone ${ }^{(1)(2)}$.

Existing methods of gesture recognition can be classified into several distinct categories, which are, view/appearance- based, model-based, space-time volume-based, and direct motion-based methods ${ }^{(3)}$. Among these methods, the template-matching approaches are simple and fast algorithms for motion analysis or recognition that can represent an entire video sequence into a single image format. For example, in the spatio-temporal template based approaches, a motion energy image (MEI) and a motion history image (MHI) are produced from an image sequence, which indicate the regions of motions, while preserving the time information of the motion ${ }^{(2),(4)}$. On the other hand, methods in Ref.(5), (6) utilize frequency domain representation of gestures, while the method in Ref. ${ }^{(7)}$ employed optical flow-based motion detection and localization.

Most of the gesture recognition methods depend on a good amount of input to train the system and the performance degrades with lesser number of available training data. However, supplying a lot of training examples may be impractical in many applications and many 


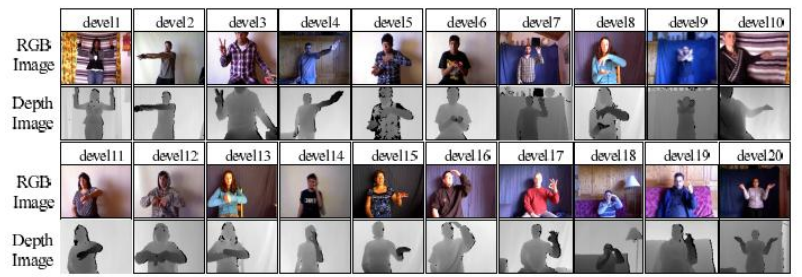

Fig. 1. Extracts from the Dataset.

consumer applications of gesture recognition will become possible only if the training is one- shot, i.e. with only one training sample for each gesture. Recently, there are a number of works that addresses the extremely difficult problem of one-shot learning gesture recognition. For example, in Ref. ${ }^{(8)}$ 3D Histogram of Flow (3DHOF) and Global Histogram of Oriented Gradient (GHOG) based features are formed from RGBD images for one-shot learning. On the other hand, authors in Ref. ${ }^{(9)}$ characterized data tensors as points on a product manifold and modeled it statistically using least squares regression. In Ref. ${ }^{(6)}$, frequency domain representation of frames and MHI are combined with statistical tools for one-shot learning gesture recognition. Some more methods of one-shot learning recognition are described in ${ }^{(10-13)}$.

\subsection{The ChaLearn Gesture Dataset}

A good number of algorithms have been developed gesture recognition using Kinect sensor by taking advantage of the depth information obtained from the mounted infrared depth sensor. Many works succeeded in identifying body parts, such as head, hand and leg, and using a sensor in tracking them to recognize actions and gestures $^{(14-16)}$. The gesture recognition process was even extended to real time application in multi-user environment for sign language recognition. The ChaLearn Gesture challenge encouraged a few researchers to attack the problem of one-shot learning gesture recognition from RGB and depth images of the Kinect sensor provided in the ChaLearn Gesture dataset. In Ref. ${ }^{(17)}$ some methods of the top contestants are discussed.

In this paper, an efficient one-shot-learning mechanism is developed specifically for the ChaLearn gesture dataset in the context of Human-Machine Interaction (HMI), which focuses on actions performed by a human who is positioned at a short distance from an infrared depth sensor. From the sequence of training depth images of human gestures the key features are extracted employing the proposed motion based template matching technique. The pre-processing step consists of back- ground removal, formation of motion history images (MHI) to track movement of the subject, development of binary images from the MHIs to obtain interest points that represent motion between frames, division of the region of interest (ROI) into several blocks and calculation of the percentage change in interest points at each block for an entire action. The feature vector is a collection of the percentage change of interest point values. The test images are mostly collections of several gestures and therefore temporal segmentation of gestures is needed. However, for simplicity, the original segmentation data provided with the dataset is used. Thus, the testing phase includes extraction of test feature vectors in the proposed way and gesture classification using correlation and Euclidean distance based classifiers. The percentage of Levenshtein distance (LD) is used as a measure of performance evaluation and a satisfactory recognition performance for a wide variety of gestures including sign languages, partially visible human figure scenarios, etc. is achieved.

\section{Proposed Method for One-Shot-Learning Gesture Recognition}

In this paper, a method of gesture recognition using one- shot-learning from a small vocabulary of gestures is developed. In the video data set, both RGB images and corresponding depth images are available. Although, training the system with only one example is difficult, the availability of depth image in addition to traditional RGB image opens doors to many new possibilities. The gestures had to be recognized from a set of gestures and matched with the vocabulary. The proposed algorithm focuses only on the depth data from the sensor for the gesture recognition model.

There are some clearly-defined hands or body or head gesture datasets, e.g., Cambridge gesture dataset ${ }^{(18)}$, Naval Air Training and Operating Procedures Standardization (NATOPS) aircraft handling signals database ${ }^{(19)}$, Keck gesture dataset ${ }^{(20)}$, Korea University Gesture (KUG) database $^{(21)}$, etc. Though all these datasets are well-known for their contents and complexities, all of them address a particular type of gestures limited to very few classes and application domains. Therefore, for the simulation purpose of the proposed method, a very rich, but extremely complicated dataset, namely, the ChaLearn Gesture Dataset, is considered in this paper ${ }^{(22)}$.

The ChaLearn Gesture database contains nine 
categories of gestures corresponding to various settings or application domains. Some frames of the video samples of the ChaLearn gesture dataset are shown in Fig. 1. Each set of data contains two types of video, a number of actions presented separately and only once for training the machine, and then different combinations of one or more of these actions performed in a sequence in each video for recognizing. In the videos, a single user is portrayed in front of a fixed camera, interacting with a computer by performing gestures. The videos are a collection of a large dataset of gestures including RGB and depth videos of 50,000 gestures recorded with the Kinect ${ }^{\mathrm{TM}}$ camera with image sizes $240 \times 320$ pixels at 10 frames per second. The videos are recorded by 20 different users and grouped in 500 batches of 100 gestures. The data is divided in different batches. Each batch including 47 sequences of 1 to 5 gestures are drawn from various small gesture vocabularies of 8 to 12 unique gestures, called a lexicon. There are over 30 different gesture vocabularies. For instance, a gesture vocabulary could consist of the signs to referee volleyball games or the signs to represent small animals in the sign language for the deaf. The complexities in this dataset are introduced by wide variation of the types of actions, environment, and position of the performer. In addition, in many cases the videos are subjected to self-occlusion. Thus, the ChaLearn Gesture Dataset is undoubtedly one of the most complex gesture datasets available to date.

\section{1 $\quad$ Feature Extraction and Training}

The depth data provided in the dataset are in RGB

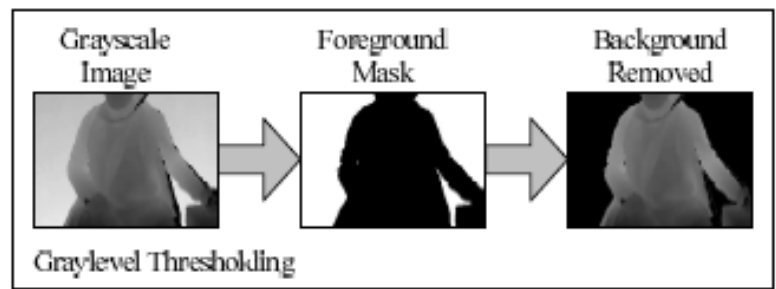

Fig. 2. Otsu's threshold to subtract background form depth image. format and therefore need to be converted to grayscale before processing. The grayscale depth data is a true representation of object distance from the camera by varying intensity of pixels from dark to bright for near too far away objects, respectively. This fact also makes it easy to employ grayscale threshold on each frame of the gesture sample to separate the foreground from the background. The threshold level is obtained by using Otsu's method ${ }^{(23)}$, which chooses the threshold to minimize the intra-class variance of the black and white pixels for making a binary image. The binary image is used as a mask to filter out the background from the depth image and thereby separate the human subject as shown in Fig. 2.

The proposed methods of gesture recognition are an outcome of the series of operations performed for feature extraction. The operations are (a) scaling down the frame sizes, (b) local MHI i.e. obtaining MHI for the last $N$ frames at each consecutive frames, (c) calculating the percentage change in $\mathrm{MHI}$ values in comparison to the total change in MHI values at each pixel, (d) dividing the resultant frame into $p \times q$ blocks, (e) calculating the percentage change in interest points (IP) at each block and, (f) performing two dimensional Fourier transform (2D-FFT) to get the final feature. The process of feature extraction from test data and classification is shown in brief in Fig. 3.

Scaling down is performed in order to reduce computational complexity. In the proposed scheme, the each frame is scaled down to $s \%$ of its original size and therefore the size of each $m \times n$ frame become mscale $\times$ nscale where mscale $=m \times s / 100$ and nscale $=n \times$ $s / 100$. In order to capture the direction of flow of motion and thereby the temporal position information of the moving parts the operation of taking the motion history image (MHI) of the lexicon is proposed. In the MHI, the silhouette sequence is condensed into grayscale images, while dominant motion information is preserved. Therefore, it can represent motion sequence in compact manner. This MHI template is also not so sensitive to silhouette noises,

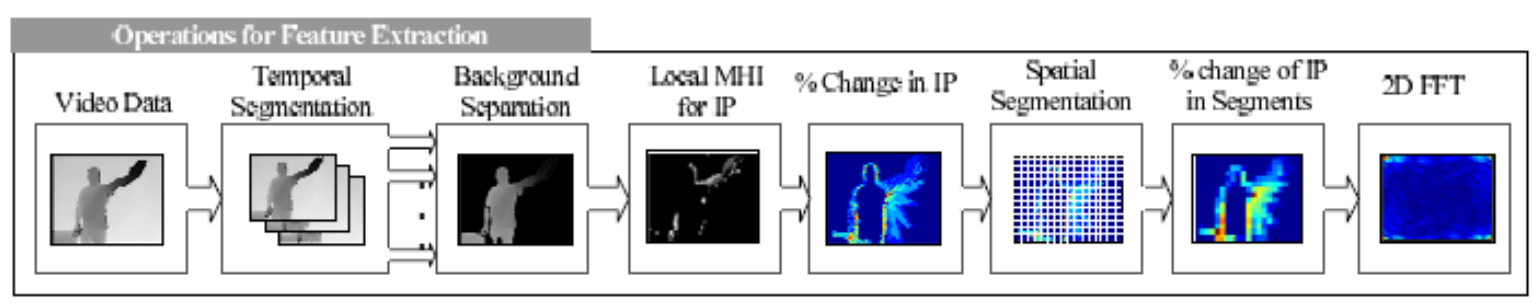

Fig. 3. Proposed method of gesture recognition. 


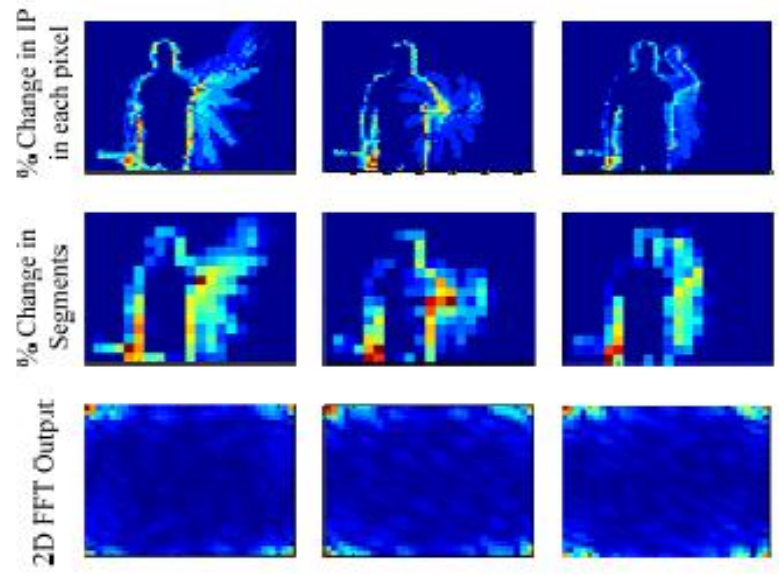

Fig. 4. Feature extraction from local MHIs.

like holes, shadows, and missing parts. These advantages make these templates as a suitable candidate for motion and gait analysis ${ }^{(24),(25)}$. Conventional MHI has the advantage of representing a range of times encoded in a single frame, and this way, the MHI spans the time scale of human gestures $^{(26)}$. It expresses the motion flow or sequence by using the intensity of every pixel in temporal manner. The motion history recognizes general patterns of movement, therefore it can be implemented with cheap cameras and lower powered CPUs ${ }^{(26)}$, in low light areas where structure cannot be easily detected. Another benefit of using the gray-scale MHI is that it is sensitive to the direction of motion and hence the MHI is better-suited for discriminating between actions of opposite directions (e.g., moving hand from left to right and from right to left) ${ }^{(27),(1)}$.

In the proposed scheme, unlike the conventional approach of taking MHI over a complete action, a local MHI of the last $N$ frames is considered. The local MHI is taken on every one of the background subtracted depth image frames of the gesture samples. To do that, first a binarized image $\Psi(x, y, t)$ for the pixel $(x, y)$ and the $t$-th frame is obtained from frame subtraction using a threshold $\phi$, i.e.,

$$
\Psi(x, y, t)=\left\{\begin{array}{ll}
1 & \text { if } \quad z(x, y, t) \geq \phi \\
0 & \text { otherwise, }
\end{array},\right.
$$

where $z(x, y, t)$ is defined using difference distance $\Delta$ as follows

$$
z(x, y, t)=\left|I_{x y}(t)-I_{x y}(t \pm \Delta)\right|,
$$

The MHI $H(x, y, t)$ can be computed from update function $\Psi(x, y, t)$ using
$H(x, y, t)=\left\{\begin{array}{l}\tau \\ \max (0, H(x, y, t-1)-\delta) \quad \text { otherwise }\end{array}\right.$,

where $\Psi(x, y, t)$ signals object presence (or motion) in the current video image, the duration $\tau$ decides the temporal extent of the movement (e.g., in terms of frames), and $\delta$ is the decay parameter. The MHI of the $t-N+1$-th frame to the $t$-th frame is taken. This update function is called for every new video frame analyzed in the sequence and MHI is computed for the last $N$ consecutive frames ${ }^{(1)}$.

The local MHI contains information related to recent movement of body parts. Therefore, the pixels with values other than zero in the local MHIs can be considered as the interest points (IP). It is to be noted that, for $N$ frame local MHI on gray scale images these pixel values can be between 255 and $255-(\mathrm{N}-1) \times \delta$. Now, if a complete action consists of $D$ number of frames, $D-N+1$ local MHI frames will be obtained. All these frames are added together and each pixel value is divided by the resultant summation of all the pixel values to be calculated the percentage change in IP at each pixel. If the $k$-th pixel at the $t$-th local MHI frame is $I P_{k}{ }^{t}$, then the overall percentage of change in IP in an action with $D-N+1$ local MHIs is

$\varphi_{\bar{i}, \bar{j}}=\frac{\sum_{i=1}^{D-N+1} I P_{k}^{i}}{\sum_{i=1}^{D-N+1} \sum_{l=1}^{m s c a l e x n s c a l e},}$,

where, $\bar{i}=1,2, \ldots$, mscale and $\bar{j}=1,2, \ldots$, nscale. The image obtained this way is an informative representation of the trend of the gesture as shown in the top row pictures of Fig. 4. Next, the resultant image is divided into $p \times q$ smaller non-overlapping blocks. Therefore, at each one of the smaller blocks the change in interest point is

$\varsigma_{\bar{k}, \bar{l}}=\sum_{i=(k-1) \text { mscalel } p)+1}^{k \times \text { mscalel } p}\left(\sum_{j=(k-1) \text { nscalel } q)+1}^{k \times n \text { scalel } q} \varphi_{i, j}\right)$,

where, $\bar{k}=1,2, \ldots, p$ and $\bar{l}=1,2, \ldots, q$. Dividing the whole image into blocks is advantageous because now the dependency of the feature on exact positioning of the body parts in a certain pixel is reduce, rather the movement performed within a block is considered. Now, for the image $\varsigma_{\bar{k}, \bar{l}}$ of size $p \times q$ the 2D discrete Fourier transform is given by, 


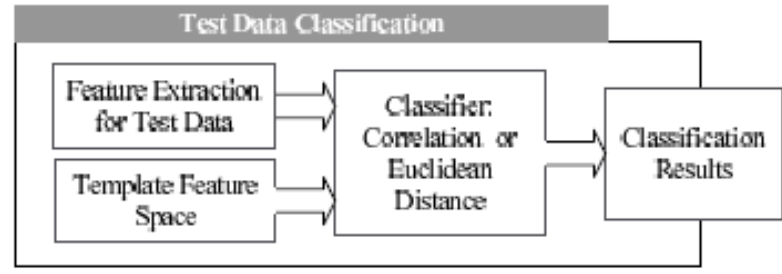

Fig. 5. Classification process flow for test data.

$$
F_{k l}(t)=\frac{1}{p q} \sum_{x=0}^{p-1} \sum_{y=0}^{q-1} \varsigma_{x y}(t) e^{-j 2 \pi\left(\frac{k x}{p}+\frac{k y}{q}\right)}
$$

where $t=1,2, \ldots, T$ and the exponential term is the basis function corresponding to each point $F_{k l}(t)$ in the Fourier space ${ }^{(28)}$. The advantage of performing Fourier transform is that when the depth values of the person are taken to the frequency domain, the position of the person becomes irrelevant, i.e., any slight movement of the camera vertically or horizontally would be nullified in the frequency response.

The above operation is performed on all the test vectors and the outcome of the $2 \mathrm{D}$ Fourier transform is utilized as feature for a particular action.

\subsection{Temporal Segmentation}

The test samples provided in the ChaLearn Gesture Dataset contain one or more gestures. Therefore, the first task is temporal segmentation of different gestures from the gesture sequences. There are a number of algorithms applied by different researchers such as ${ }^{(6),(9)}$ for finding the temporal segment boundaries. The test samples provided in the ChaLearn Gesture Dataset contain one or more gestures. Therefore, the first task is to separate the different gestures from the gesture sequences. However, in order to focus solely on the gesture recognition task, the temporal segment positions provided with the ChaLearn Gesture Dataset by the developers is utilized directly for separating different actions from a single video file.

\subsection{Testing and Classification}

After extracting the features from the gestures in the training dataset of a particular lexicon, a feature vector table is formed for that lexicon as shown in Fig. 3. Then, for the test samples, first temporal segmentation is done if multiple gestures exist. Then features are extracted for each gesture in a manner similar to that done for training samples. The testing and classification phase is represented with a block diagram in Fig. 5. One of the classifiers used is the correlation based distance measure. The correlation coefficient is calculated between the features obtained from the test gesture to that similar feature obtained from each of the training gestures. The coefficient of correlation, $r$, is a mathematical measure of how much one value is expected to be influenced by change in another one. It is a widely used measure for image and gesture recognition ${ }^{(28)}$. The correlation coefficient between two images $A$ and $B$ is defined as,

$$
r=\frac{\sum_{k=0}^{m-1} \sum_{l=0}^{n-1}\left(A_{k l}-\bar{A}\right)\left(B_{k l}-\bar{B}\right)}{\sqrt{\sum_{k=0}^{m-1} \sum_{l=0}^{n-1}\left(A_{k l}-\bar{A}\right)^{2}} \sqrt{\sum_{k=0}^{m-1} \sum_{l=0}^{n-1}\left(B_{k l}-\bar{B}\right)^{2}}},
$$

where, $A_{k l}$ is the intensity of pixel $(k, l)$ in image $A$ and $B_{k l}$ is the intensity of pixel $(k, l)$ in image $B . A$ and $B$ are the mean intensity of all the pixels of image $A$ and $B$, respectively. If $r= \pm 1$ then there is a strong positive/negative correlation between the two images, i.e., they are identical/negative of one other. If $r$ is zero then there is no correlation among the matrices. A high value of the correlation coefficient between the same features of the test sample with that of one of the training samples indicates a higher probability of the two gestures to be identical and vice versa for a low value of correlation coefficient.

Another measure of classification is the well-practiced Euclidean distance (ED) measure in which the point-by-point sum square distance between a testing feature and all the training features is calculated. The training feature for which minimum ED is obtained is considered to be the similar action as the test data.

\section{Experimental Results}

For the purpose of evaluating gesture recognition performance of the proposed method, the Levenshtein distance (LD) measure is employed. The Levenshtein distance, also known as edit distance, is number of deletions, insertions, or substitutions required matching an array with another ${ }^{(29)}$. The LD measure has a wide range of applications, such as spell checkers, correction systems for optical character recognition and other similar systems ${ }^{(30)}$, (31). For the proposed gesture recognition system, if the list of labels of true gesture in a test sequence is $T$, while the labels corresponding to the recognized gestures for the same sequence is $R$, then the Levenshtein distance $L(R, T)$ will represent the minimum number of edit operations that one has to perform to go from $R$ to $T$ (or vice versa). The evaluation criteria is the percentage $L D$ which is the sum of 


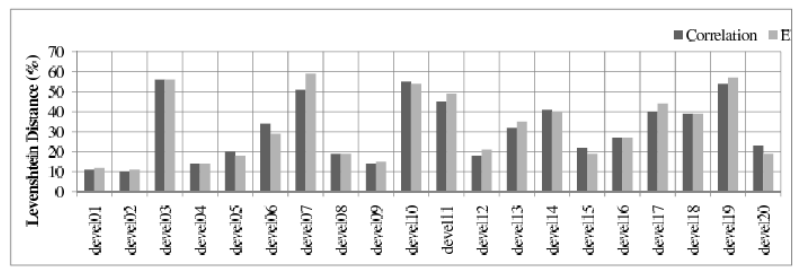

Fig. 6. Levenshtein Distances (\%) for different lexicons.

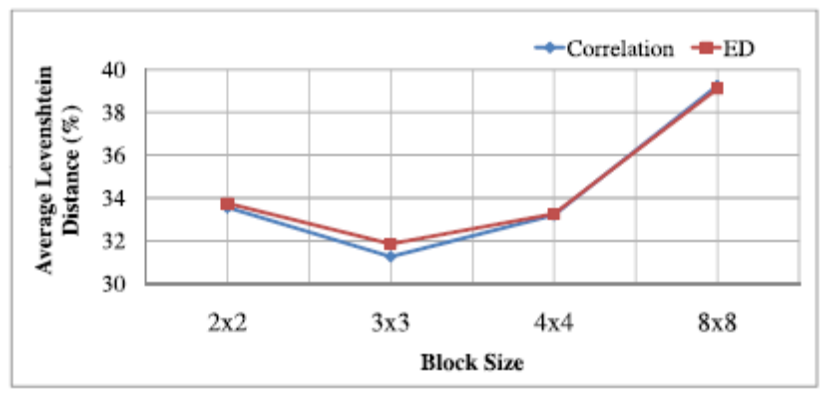

Fig. 7. Levenshtein Distances (\%) for different block sizes (image scaled to $20 \%$ ).

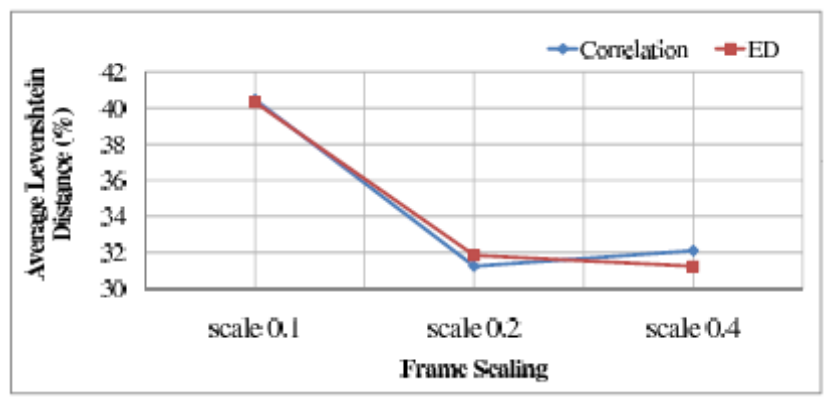

Fig. 8. Levenshtein Distances (\%) for different scales (block size $3 \times 3$ ).

all the LDs obtained from a lexicon divided by the total number of true gestures in that lexicon and multiplied by 100. It is evident that the higher the value of $L D$, the more is the number of wrong estimations. Thus a lower value of LD is expected ${ }^{(29)}$.

For the purpose of analysis, the first 20 lexicons, namely Devel01 to Devel20 from CGD2011s ${ }^{(22),(32)}$ dataset are considered. In this dataset, different actions in a set are assigned to a number as a label and a string containing the labels of actions are provided for each gesture sequence.

The classification results obtained in terms of percentage of Levenshtein Distance (LD) using correlation and ED based classifiers by employing the proposed method is shown in Fig.6 for each lexicon of the first 20 development data. It can be seen from the figure that the result varies widely from set to set. The wide variation is
Table 1. Performance Comparison.

\begin{tabular}{cl}
\hline Methods & $\%$ LD \\
\hline Correlation(Proposed) & $31.25 \%$ \\
\hline ED(Proposed) & $31.85 \%$ \\
\hline 3DHOF+GHOF & $43.32 \%$ \\
\hline Template Matching & $62.56 \%$ \\
\hline Dynamic Time Wraping & $49.41 \%$ \\
\hline BoVW & $72.32 \%$ \\
\hline
\end{tabular}

caused by the different types of motions in different sets. In some sets (devel03, devel07, devel10, devel19), percentage of $\mathrm{LD}$ is higher than the rest because the gestures of these sets are mostly hand gestures similar to sign language, which are very difficult to differentiate. Also, in many samples same gestures are performed in different positions relative to the body. Therefore the feature extracted by the MHI is different for the same gesture in different sequences and hence, can hardly be matched with the original training gesture. The performances of the proposed method are also limited by the accuracy of separation of different actions from the videos and unexpected movements of the performer. Also in some videos, the performer changed position while performing a gesture which added some unexpected features in the model that gave a wrong match. So the result might have been improved if these could be overcome. Also, in Fig. 6, it can be seen that better classification result is obtained for correlation classifier rather than ED in most cases. It can be expected that more advanced classifiers may produce even better results.

In Figs. 8 and 7 the effect of the scaling factor and the smaller block sizes on the performance of the proposed scheme are depicted. It can be seen from these figures that best performance is achieved for a scaling factor of 0.2 , i.e., scaling to $20 \%$ of the original image, combined with a block size of $3 \times 3$.

The performance of the proposed method is compared with a number of other methods proposed by several researchers in Table I. Simple 3DHOF+GHOG descriptors proposed by ${ }^{(8)}$ utilize 3D Histogram of Flow (3DHOF) and Global Histogram of Oriented Gradient (GHOG). In the Template Matching technique the average of all depth frames for each action is used as the descriptor. The test video is split in slices estimated using the average size of actions. In recognition phase each slice of the video is compared with all the templates. The proposed method is also compared with Dynamic Time Warping (DTW) with $3 \mathrm{DHOF}+$ GHOG features ${ }^{(33),(8)}$. Another renowned method 
is the Bag of Visual Words (BoVW) ${ }^{(34)}$. It can be seen from the table that the recognition accuracy of the proposed method outperforms all the other methods in terms of LD.

\section{Conclusions}

In this paper, a novel approach for one-shot learning gesture recognition employing combinations of local MHI, statistical measures and frequency domain transformation on motion depth images is proposed. The proposed method first scale down the frame size and subtracts the background by employing grayscale threshold. Then, features from each gesture sequence are extracted based on percentage change of interest points across all local MHIs, special segmentation of the change in IP in smaller blocks and finally two dimensional Fourier transform of the resultant segmented representation. Correlation based and Euclidean distance based classifiers are employed to classify gestures in this multi-class recognition problem. The dataset, namely, the ChaLearn Gesture Dataset 2011, targeted for experimental evaluation is a very rich, but difficult dataset to handle having a lot of variation and classes. This fact presented unprecedented obstacles which had to be overcome systematically with different methods. Through extensive evaluation it is proved that the proposed combination of features can provide a superior recognition performance in comparison to few other methods for one of the most complex dataset of gestures to date.

\section{References}

(1) M. A. R. Ahad, Computer Vision and Action Recognition: A Guide for Image Processing and Computer Vision Community for Action Understanding, ser. Atlantis Ambient and Pervasive Intelligence. Atlantis Press, 2011.

(2) M. A. R. Ahad, J. Tan, H. Kim, and S. Ishikawa, "Human activity recognition: Various paradigms," in Control, Automation and Systems, 2008. ICCAS 2008. International Conference on, pp. 1896- 1901, 2008.

(3) M. A. R. Ahad, J. K. Tan, H. Kim, and S. Ishikawa, "Motion history image: its variants and applications," Mach. Vision Appl., vol. 23, no. 2, pp. 255-281, 2012.

(4) A. F. Bobick and J. W. Davis, "The recognition of human movement using temporal templates," IEEE Trans. Pattern Anal. Mach. Intell., vol. 23, no. 3, pp. 257-267, Mar. 2001.

(5) H. Imtiaz, U. Mahbub, and M. A. R. Ahad, "Action recognition algorithm based on optical flow and RANSAC in frequency domain," in SICE Annual Conference (SICE), 2011 Proceedings of, pp. 16271631, 2011.

(6) U. Mahbub, H. Imtiaz, T. Roy, M. S. Rahman, and M. A. R. Ahad, "A template matching approach of one-shot-learning gesture recognition," Pattern Recognition Letters, 2012. [Online]. Available:http://www.sciencedirect.com/science/article /pii/S0167865512002991

(7) U. Mahbub, H. Imtiaz, and M. A. R. Ahad, "An optical flow based approach for action recognition," in Computer and Information Technology (ICCIT), 2011 14th International Conference on, pp. 646-651, 2011.

(8) S. R. Fanello, I. Gori, G. Metta, and F. Odone, "One-shot learning for real-time action recognition," To appear in Iberian Conference on Pattern Recognition and Image Analysis (IbPRIA), 2013.

(9) Y. M. Lui, "Human gesture recognition on product manifolds," Journal of Machine Learning Research, vol. 13, pp. 3297-3321, 2012.

(10)L. Fei-Fei, R. Fergus, and P. Perona, "One-shot learning of object categories," Pattern Analysis and Machine Intelligence, IEEE Transactions on, vol. 28, no. 4, pp. $594-611,2006$.

(11) B. Lake, R. Salakhutdinov, J. Gross, and J. Tenenbaum, "One shot learning of simple visual concepts," in Proceedings of the $33^{\text {rd }}$ Annual Conference of the Cognitive Science Society, 2011.

(12) W. Yang, Y. Wang, and G. Mori, "Human action recognition from a single clip per action," MLVMA, pp. 482-489, 2009.

(13) H. J. Seo and P. Milanfar, "Action recognition from one example,” IEEE Trans. Pattern Anal. Mach. Intell., vol. 33, no. 5, pp. 867-882, 2011.

(14) M. Tang, "Recognizing hand gestures with Microsoft's Kinect," Computer, vol. 14, no. 4, pp. 303-313, 2011.

(15) Z. Ren, J. Yuan, and Z. Zhang, "Robust hand gesture recognition based on finger-earth mover's distance with a commodity depth camera," in Proceedings of the 19th ACM international conference on Multimedia, pp. 1093-1096, 2011.

(16) R. Zhou, J. Meng, and J. Yuan, "Depth camera based hand gesture recognition and its applications in human-computer-interaction," in $8^{\text {th }}$ International Conference on Information, Communications and Signal Processing (ICICS), pp. 1-5, 2011.

(17) I. Guyon, V. Athitsos, P. Jangyodsuk, B. Hammer, and 
H. J. E. Balderas, "ChaLearn gesture challenge: Design and first results," CVPR workshop, pp. 1-6, 2012.

(18) T.-K. Kim and R. Cipolla, "Canonical correlation analysis of video volume tensors for action categorization and detection," IEEE Trans. Pattern Anal. Mach. Intell., vol. 31, no. 8, pp. 1415-1428, 2009.

(19) Y. Song, D. Demirdjian, and R. Davis, “Tracking body and hands for gesture recognition: NATOPS aircraft handling signals database." in IEEE Conference on Automatic Face and Gesture Recognition, pp. 500-506, 2011.

(20) Z. Jiang, Z. Lin, and L. Davis, "Recognizing human actions by learning and matching shape-motion prototype trees," IEEE Trans. Pattern Anal. Mach. Intell., vol. 34, no. 3, pp. 533-547, 2012.

(21) B.-W. Hwang, S. Kim, and S.-W. Lee, "A full-body gesture database for automatic gesture recognition," in IEEE Conference on Automatic Face and Gesture Recognition, pp. 243-248, 2006.

(22) "ChaLearn Gesture Dataset (CGD2011)," ChaLearn, California, 2011.

(23) N. Otsu, "A threshold selection method from gray-level histograms," IEEE Trans. on Systems, Man, and Cybernetics, vol. 9, no. 1, pp. 62-66, 1979.

(24) L.-F. Liu, W. Jia, and Y.-H. Zhu, "Survey of gait recognition," in Proceedings of the intelligent computing 5th International Conference on Emerging Intelligent Computing Technology and Applications, pp. 652-659, 2009.

(25) J. Liu and N. Zhang, "Gait history image: a novel temporal template for gait recognition," Proc. IEEE International Conf. on Multimedia and Expo, pp. 663-666, 2007.

(26) G. R. Bradski and J. W. Davis, "Motion segmentation and pose recognition with motion history gradients," Mach. Vision Appl., vol. 13, no. 3, pp. 174-184, Jul. 2002.

(27)A. Bobick and J. Davis, "An appearance-based representation of action," in Proceedings of the 1996 International Conference on Pattern Recognition (ICPR), pp. 307-312, 1996.

(28) R. C. Gonzalez and R. E. Woods, Digital Image Processing, 2nd ed. Boston, MA, USA: Addison-Wesley Longman Publishing Co., Inc., 2001.

(29) V. I. Levenshtein, "Binary codes capable of correcting deletions, insertions, and reversals," Soviet Physics Doklady, vol. 10, no. 8, pp. 707-710, 1966.
(30) J. D. Golic and M. J. Mihaljevic, "A generalized correlation attack on a class of stream ciphers based on the levenshtein distance," Journal of Cryptology, vol. 3, pp. 201-212, 1991.

(31) A. Marzal and E. Vidal, "Computation of normalized edit distance and applications," IEEE Trans. Pattern Analysis and Machine Intelligence, vol. 15, no. 9, pp. 926-932, 1993.

(32) "ChaLearn Gesture Dataset. [online]," ChaLearn, California, http://www.kaggle.com/c/GestureChallenge

(33) H. Sakoe and S. Chiba, "Dynamic programming algorithm optimization for spoken word recognition," Acoustics, Speech and Signal Processing, 1978.

(34) D. Wu, F. Zhu, and L. Shao, "One shot learning gesture recognition from rgbd images," CVPR Workshop on Gesture Recognition, 2012. 